\title{
COVID-19 in a remote First Nations community in British Columbia, Canada: an outbreak report
}

\author{
Courtney R. Smith MPH, Charmaine Enns MD, Dan Cutfeet MD, Shannon Alfred, Nicole James BScN, \\ Jennifer Lindbeck MSc, Shannon Russell PhD
}

Abstract

Background: In April 2020, British Columbia experienced its first outbreak of COVID-19 in a remote First Nations community. The objective of this paper was to describe the outbreak, including epidemiological and laboratory findings, and the public health response.

Methods: This report summarizes an outbreak of COVID-19 on Cormorant Island, British Columbia, in March and April 2020. Confirmed cases underwent investigation and contact tracing. Supports were provided to ensure successful isolation and quarantine for cases and contacts. Messaging to the community was circulated by trusted community members. Descriptive and social network analyses were conducted to describe the outbreak as it evolved. All case specimens underwent whole-genome sequencing.

Results: Thirty cases of SARS-CoV-2 infection were identified. Those infected had a median age of 34 years (range 15-77), and the majority identified as female $(19,63 \%)$ and as First Nations $(27,90 \%)$. The most common symptoms included chills, cough, diarrhea, headache and fever. Five people were hospitalized (17\%) and 1 died (3\%). Percent positivity in the community was $18 \%$. Transmission occurred primarily during evening social gatherings and within households. Two weeks after control measures were initiated, no further cases were identified. All cases were genetically related by 2 single nucleotide polymorphisms or fewer, and they belonged to the most dominant SARS-CoV-2 lineage present in British Columbia in April 2020.

Interpretation: A community-led response was essential for the effective containment of this outbreak that included 30 cases, preventing onward transmission of the virus. Lessons learned from the management of this outbreak can inform response to other similar outbreaks in First Nations communities across Canada.

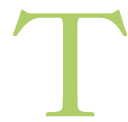
he first case of COVID-19 in Canada was recorded in late January 2020. ${ }^{1}$ Two months later, in March 2020, community transmission was documented, and the pandemic took hold across the country. As of November 4, 2021, Canada has had more than 1.7 million cases of COVID-19, and just over 29000 deaths. ${ }^{2}$ The province of British Columbia has had more than 204000 cases and more than 2000 deaths. ${ }^{3}$ The impact on the population at large has been substantial, but the impact on vulnerable communities has been even more pronounced. In particular, remote First Nations communities are at higher risk of the spread of SARS-CoV-2 as a result of highdensity households, limited transportation options, and limited medical facilities and services. ${ }^{4,5}$ These communities might also be at increased risk for severe outcomes due to the higher prevalence of chronic disease. ${ }^{4,5}$ Historic mistrust in government may be a compounding factor, causing people to delay accessing medical services. ${ }^{5,6}$ Increases in SARS-CoV-2 transmission in First Nations communities during Canada's second wave of the pandemic seemed to confirm initial concerns, resulting in calls for multidimensional and culturally competent outbreak response. ${ }^{5}$
In March 2020, British Columbia experienced its first outbreak of COVID-19 in a remote First Nations community on Cormorant Island. As the first remote First Nations outbreak of its kind in the province and one of the first in Canada, the outbreak presented challenges and opportunities for public health response. The objective of this report was to describe the outbreak that occurred in March and April 2020, including epidemiological and laboratory findings and the public health response. Our goal was to inform action in similar communities in the coming months (and perhaps years) both in Canada and abroad.

\section{Competing interests: None declared.}

This article has been peer reviewed.

Correspondence to: Courtney Smith, courtney.r.smith@phac-aspc.gc.ca

CMAJ Open 2021 November 30. DOI:10.9778/cmajo.20210054 


\section{Methods}

\section{Study design}

This article reports on an outbreak of COVID-19 on Cormorant Island in British Columbia in March and April 2020. Outbreak response was a collaboration between 'Namgis First Nation and Island Health; both entities are represented in the authorship of this paper.

\section{Setting and population}

Cormorant Island is located off the northeast coast of Vancouver Island, British Columbia. It is home to First Nations and non-First Nations residents, who live on-reserve (49\%) or off-reserve (51\%). ${ }^{7}$ The community consists of approximately 1000 residents and is accessible only by air and boat.

On Apr. 11, 2020, the first confirmed case of COVID-19 was reported in a resident of Cormorant Island. On Apr. 14, 2020, intensive testing for SARS-CoV-2 began for all symptomatic members of the community. By Apr. 17, 2020, the case count had reached 8 and a state of emergency was declared. On Apr. 20, 2020, ferry travel was restricted to essential travel only, and on Apr. 21, 2020, a nightly curfew was established using the local tsunami siren.

\section{Case definition}

A confirmed case of COVID-19 was defined as a person with laboratory confirmation of SARS-CoV-2 infection with a sample collected via nasopharyngeal swab; testing performed at a community, hospital or reference laboratory running a validated assay; and detection of at least 1 specific gene target by a nucleic acid amplification test assay (e.g., realtime polymerase chain reaction or nucleic acid sequencing). ${ }^{8}$ The index case was the confirmed case with the earliest date of symptom onset.

\section{Data sources}

The provincial COVID-19 case report form was used to conduct case investigations, ${ }^{9}$ and all data were entered into the provincial public health information system, Panorama. Information extracted from the case report form included case identifiers, demographic characteristics, risk factors, exposures and symptoms. All data were verified for accuracy. Daily phone calls took place between the epidemiologist, the communicable disease nurse and the community health nurse to relay information about contact tracing. Information about hospitalization status was acquired from the electronic medical records system (Island Health). Clinical outcomes were considered over a 3-month period.

\section{Case-finding}

From Apr. 14, 2020, to May 9, 2020, a drive-through testing centre provided community access to testing for 6 hours a day ( 9 am to $3 \mathrm{pm}$ ), 7 days a week. Testing was by appointment and was booked through a central call-in number. Select testing was also provided at residents' homes for those unable to travel to the testing centre. Availability for testing continued after May 9, 2020, for 1.5 hours a day (10 am to 11:30 am).
Testing was recommended for all people who were experiencing symptoms related to COVID-19, including fever, chills, cough, shortness of breath, sore throat, pain with swallowing, runny nose, nasal congestion, loss of sense of smell, headache, muscle aches, fatigue or loss of appetite. ${ }^{8}$ Messaging about the availability of testing and recommendations that people get tested were circulated via the community Facebook page by trusted community members, including the senior community physician, the community nurse and the 'Namgis First Nation Chief.

\section{Laboratory testing}

All nasopharyngeal swabs were sent to the Island Health Laboratory in Victoria, B.C., to undergo polymerase chain reaction testing for SARS-CoV-2. Specimens were also forwarded to the B.C. Centre for Disease Control (BCCDC) Public Health Laboratory for whole-genome sequencing. Nucleic acids were extracted using a MagMAX instrument (Thermo Fisher). Whole-genome sequencing of SARS-CoV-2 was performed using the ARCTIC protocol (version 1.1.3) and a MinION sequencer (Oxford Nanopore Technologies). The amplification primers and sequencing method have been described in detail elsewhere. ${ }^{10}$ High-quality sequences were assigned a SARS-CoV-2 lineage based on the Phylogenetic Assignment of Named Global Outbreak Lineages tool (Pangolin; version 1.1.14). ${ }^{11}$ Phylogenetic analysis was performed using Nextstrain (Augur version 10.0.0 and Auspice version 2.18.1). ${ }^{12}$ Whole-genome sequencing results were accessed on Oct. 1, 2020, and compared with province-wide case and outbreak data using the BCCDC SARS-CoV-2 whole-genome sequencing database in conjunction with the BCCDC Public Health Laboratory Operations Viewer and Reporter.

\section{Case investigation}

All confirmed cases underwent case investigation and contact tracing over the phone using the provincial COVID19 case report form, ${ }^{9}$ conducted by a communicable disease nurse. A close contact was defined as a person who provided direct care for the case or who had other similar close physical contact without consistent and appropriate use of personal protective equipment; lived with the case or had face-to-face contact within $2 \mathrm{~m}$ for more than 15 minutes (could be cumulative); or had direct contact with the infectious body fluids of a confirmed case without wearing recommended personal protective equipment. ${ }^{8}$

The start of the infectious period for contact tracing was defined as 48 hours before symptom onset. If cases could not define the date of symptom onset, their infectious period was calculated based on the date of testing. ${ }^{8}$ When contact tracing was challenging, the community health nurse provided assistance, given their role as a trusted community member. The nurse was also able to provide context for social connections in the community that were not always captured through case interviews. 


\section{Case and contact monitoring}

Confirmed cases were asked to isolate from others for 10 days after symptom onset. ${ }^{8}$ Close contacts were asked to quarantine for 14 days from their last known exposure to a case. ${ }^{8}$ Daily monitoring for cases and contacts was available via online survey, a phone call or a home visit from the local community health nurse. The method of monitoring was determined on a case-by-case basis, considering the needs of each case and contact. The online survey was developed by a telecom company in collaboration with Island Health and included daily questions about temperature, shortness of breath, cough, chills, sore throat, congestion, headaches, muscle aches, abdominal pain, nausea or vomiting, diarrhea, fatigue, confusion and conjunctivitis. If a case or contact reported a new or worsening symptom in their daily online survey, they received a follow-up call from a communicable disease nurse.

Contacts who developed symptoms during their quarantine period were referred for testing. To support successful isolation and quarantine for cases and contacts, various resources were mobilized based on individual needs, including phones, credits for phone minutes, tablets, pulse oximetry, alternative housing for those unable to isolate adequately or quarantine away from others, and a community-managed alcohol program for those with dependencies.

When necessary, arrangements were made to transfer cases to off-island hospitals for medical treatment. A low threshold was encouraged for medical transfers off the Island. Community cases were considered recovered if 10 days had passed since symptom onset with resolution of fever and improvement of symptoms. ${ }^{8}$

\section{Data analysis}

We used Excel (Microsoft) and SAS (SAS Institute) to summarize the epidemiological data. We used descriptive statistics to examine the demographics of the outbreak and its severity. We conducted social network analysis in Pajek (http://mrvar. fdv.uni-lj.si/pajek/) to visualize transmission in the community. Social network analyses were updated once per day throughout the outbreak to inform public health decisionmaking and evaluate the completeness of the contact tracing. Social network analyses included confirmed cases and their close contacts.

\section{Ethics approval}

As an outbreak report, this work did not require formal research ethics review. This work has been completed with the full knowledge and participation of 'Namgis First Nation. It is with the consultation and permission of 'Namgis First Nation that this report is being published.

\section{Results}

A total of 30 confirmed cases of COVID-19 were identified, for a cumulative incidence of 3144 per 100000 population $(n=954)$. Dates of symptom onset ranged from Mar. 23, 2020, to Apr. 28, 2020 (Figure 1). The median age of cases was 34 years (range 15-77; Table 1). A majority of cases were female $(n=19,63 \%)$, and almost all identified as First Nations $(n=27,90 \%)$. Five cases $(n=5,17 \%)$ were hospitalized, and $1(n=1,3 \%)$ died as a result of COVID-19.

Length of hospital stay varied from 5 to 41 days (median 9 days). Of the 5 hospitalized cases, 2 (40\%) were admitted to

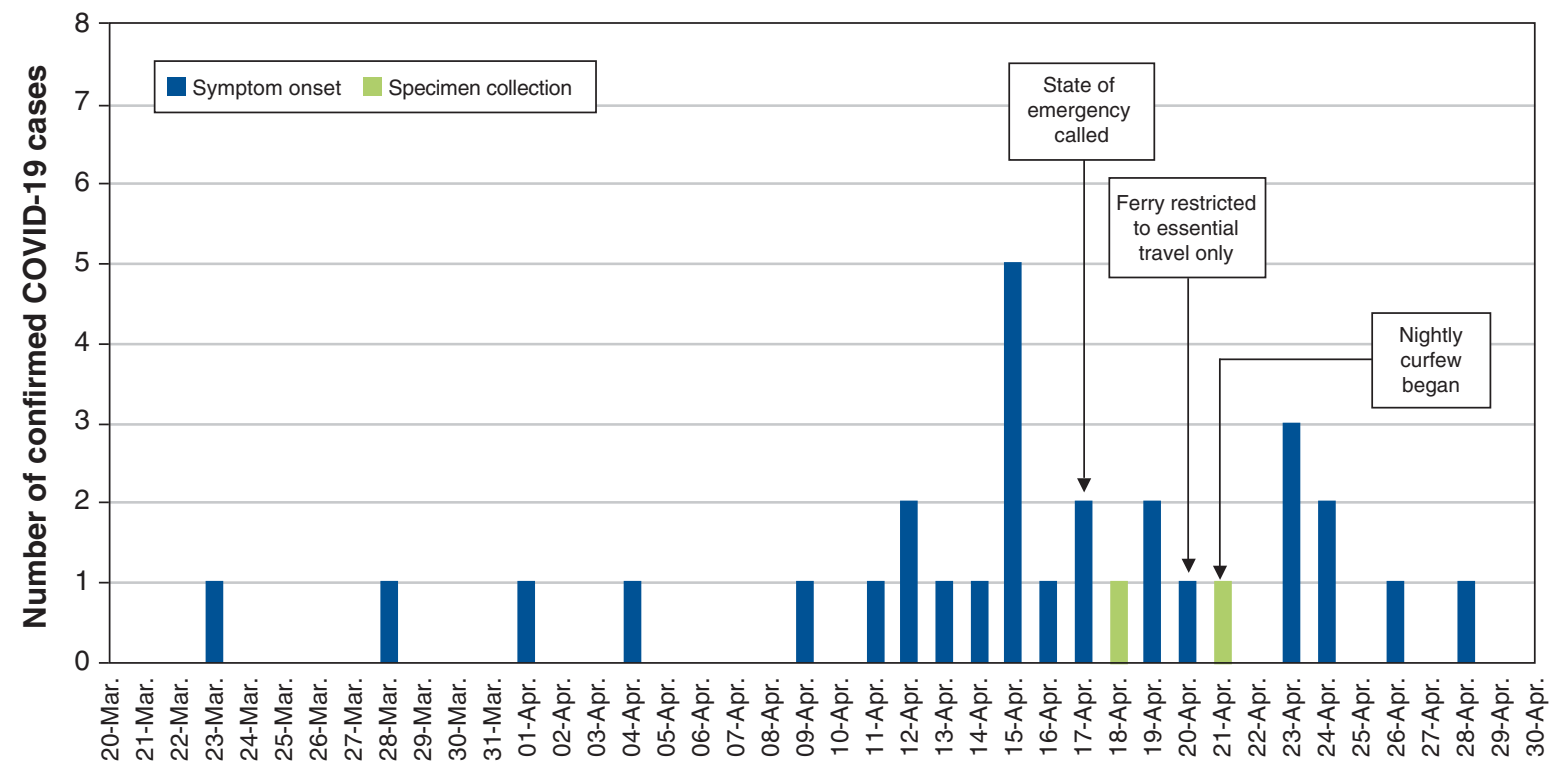

Episode date

Figure 1: Epidemic curve for confirmed COVID-19 cases, March to April 2020, by date of symptom onset or specimen collection. The state of emergency ended on May 9, 2020, and the outbreak was declared over on May 26, 2020. 
an intensive care unit. The most predominant symptoms included chills $(n=24,80 \%)$, cough $(n=21,70 \%)$, diarrhea $(n=21,70 \%)$, headache $(n=20,67 \%)$ and fever $(n=17,57 \%)$.

Transmission occurred primarily via 2 social gatherings and in 3 households (Figure 2). The 2 social gatherings, which occurred on Apr. 9 and Apr. 12, 2020, were associated

\begin{tabular}{|c|c|}
\hline Characteristic & No. $(\%)$ of cases \\
\hline \multicolumn{2}{|l|}{ Age, yr } \\
\hline Mean \pm standard deviation & $38 \pm 18.5$ \\
\hline Median (range) & $34(15-77)$ \\
\hline \multicolumn{2}{|l|}{ Sex } \\
\hline Female & $19(63)$ \\
\hline Male & $11(37)$ \\
\hline \multicolumn{2}{|l|}{ First Nations status } \\
\hline First Nations & $27(90)$ \\
\hline Non-First Nations & $2(7)$ \\
\hline Unknown & $1(3)$ \\
\hline \multicolumn{2}{|l|}{ Hospitalization for COVID-19 } \\
\hline Ever hospitalized & $5(17)$ \\
\hline Never hospitalized & $25(83)$ \\
\hline \multicolumn{2}{|l|}{ Clinical outcome } \\
\hline Recovered & $29(97)$ \\
\hline Deceased & $1(3)$ \\
\hline \multicolumn{2}{|l|}{ Symptoms } \\
\hline Chills & $24(80)$ \\
\hline Cough & $21(70)$ \\
\hline Diarrhea & $21(70)$ \\
\hline Headache & $20(67)$ \\
\hline Fever & $17(57)$ \\
\hline Rhinorrhea & $15(50)$ \\
\hline Myalgia & $13(43)$ \\
\hline Shortness of breath or breathing difficulty & $11(37)$ \\
\hline Nausea & $10(33)$ \\
\hline Weakness & $10(33)$ \\
\hline Arthralgia & $8(27)$ \\
\hline Pharyngitis & $7(23)$ \\
\hline Fatigue & $7(23)$ \\
\hline Conjunctivitis & $6(20)$ \\
\hline Irritability & $4(13)$ \\
\hline Hypotension & $3(10)$ \\
\hline Tachypnea & $3(10)$ \\
\hline Vomiting & $3(10)$ \\
\hline Confusion & $2(7)$ \\
\hline Nasal congestion & $2(7)$ \\
\hline Acute respiratory distress syndrome & $0(0)$ \\
\hline
\end{tabular}

with 18 of the $30(60 \%)$ confirmed cases when considering primary and secondary cases. The 3 households had 2-5 cases each. Several components of the social network analysis remained unconnected after contact tracing was finished, demonstrating that the contact tracing was incomplete. Two weeks after the state of emergency was declared, no further cases were identified.

The investigation did not determine how SARS-CoV-2 was introduced into the community. The index case did not report any travel off-island during their exposure period, although they did work in a setting where they may have been exposed to people with recent off-island travel.

The state of emergency ended on May 9, 2020, and the outbreak was declared over on May 26, 2020, 2 incubation periods after the last date of symptom onset (Apr. 28, 2020).

\section{Laboratory findings}

At the time the outbreak was declared over, $16 \%$ of the population had been tested for SARS-CoV-2 $(n=153)$, and overall test positivity was $18 \%$. Twenty-one cases generated highquality whole-genome sequencing data, 5 cases generated partial sequence data and 4 cases failed. The Pangolin lineage designation for all cases in the outbreak was B.1, the most dominant SARS-CoV-2 lineage identified from British Columbia in April 2020. All of the sequenced cases were considered genetically related by 2 single nucleotide polymorphisms (SNPs) or fewer (Figure 3).

Two clusters of cases were considered genetically identical, and these clusters differed by a single SNP (Figure 3). The cluster of 4 identical cases (Cluster A) was genetically identical to cases in British Columbia that were epidemiologically linked to the Pacific Dental Conference, a large superspreading event in Vancouver that occurred from Mar. 5-7, 2020. The descendants of this cluster that were detected only on Cormorant Island were not transmitted any further in British Columbia after the outbreak was declared over, based on the over 3000 genomes that had been successfully sequenced by the BCCDC Public Health Laboratory as of Oct. 1, 2020. The SARS-CoV-2 sequences in this outbreak differed from the reference strain, MN908947.3 (Wuhan-Hu-1), by 6-8 SNPs.

\section{Interpretation}

Over the course of this outbreak, 30 confirmed cases of COVID-19 were identified, with a cumulative incidence of 3144 per 100000 population. At the same time point, the province of British Columbia had a cumulative incidence of 49.7 per $100000 . .^{13}$ Even 7 months later, at the height of Canada's second wave in December 2020, Cormorant Island's cumulative incidence far surpassed that of both British Columbia (860 per 100000) and Canada (1310 per 100000$).^{2}$ In line with these findings, percent positivity for the same time period was also much higher for Cormorant Island (18\%) than for Canada overall $(7 \%)$. $^{2}$ During the first wave of the COVID-19 pandemic, Canadian First Nations communities fared better than non-First Nations population overall $;{ }^{14}$ the outbreak summarized here presents an important exception. 

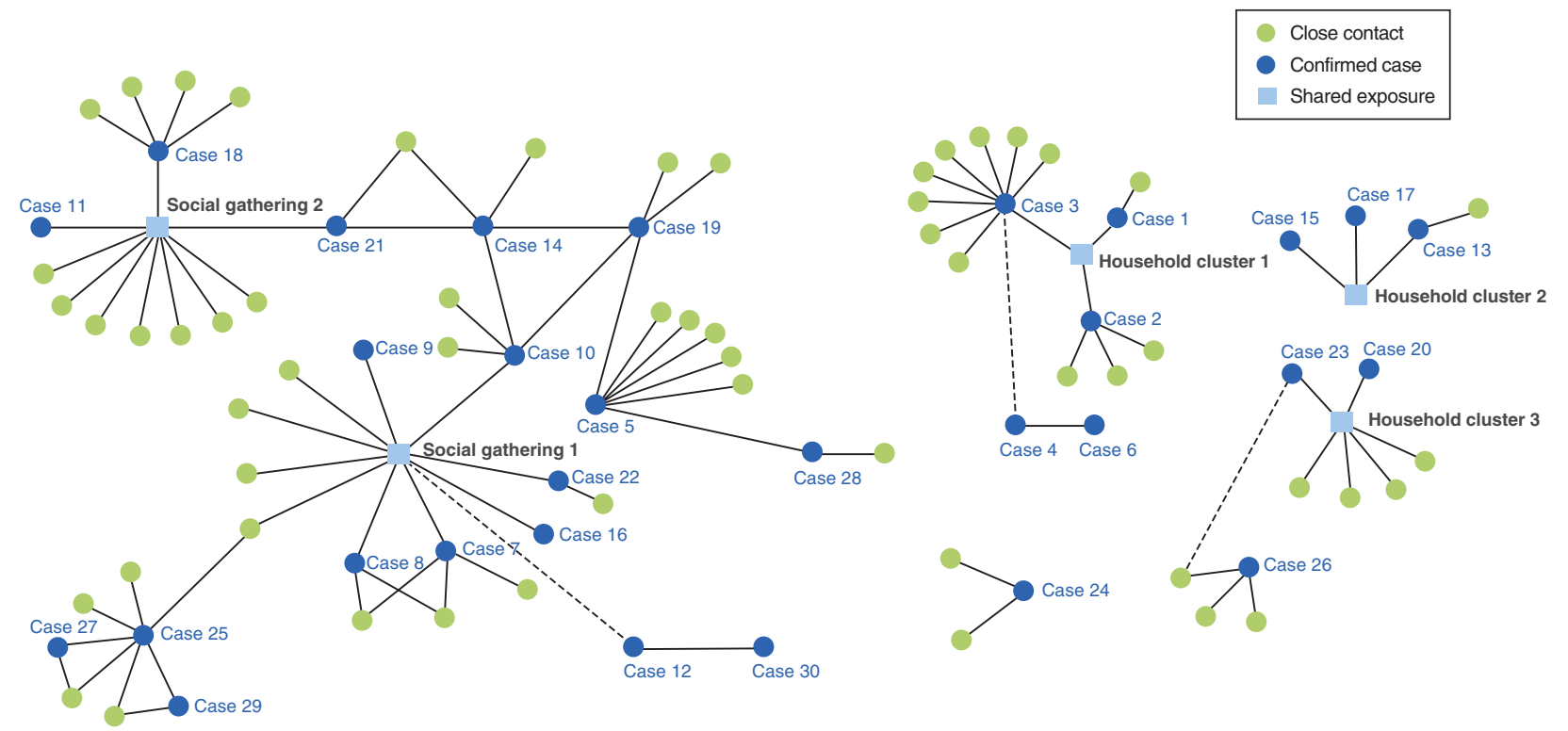

Figure 2: Social network analysis for confirmed COVID-19 cases and their contacts. Solid lines indicate a known connection between individuals, determined through contact tracing. Dotted lines indicate a potential connection based on known relationships, but one that could not be confirmed via contact tracing.

The case fatality rate and proportion of cases hospitalized for Cormorant Island (3\% and 17\%, respectively) were similar to those reported for Canada overall in April 2020. ${ }^{15}$ However, the focus on symptomatic testing in Cormorant Island, the low threshold for medical transfers and the inclusion of asymptomatic cases in the Canadian calculations may mean that these values are not comparable. Notably, although the population of Cormorant Island is fairly evenly split between First Nations and non-First Nations individuals, the First Nations community was disproportionately affected. Trends of increased morbidity have been identified in Indigenous communities around the world during previous pandemics, and it has been suggested that social and health inequities resulting from colonialism were contributing factors. ${ }^{16} \mathrm{We}$ need to listen and respond to Indigenous communities as they define health, and to inform the collective actions needed to address and minimize inequities.

The results of the social network analysis were used to inform public health action based on transmission patterns, and to provide real-time evaluation of the completeness of contact tracing. Social network analysis of this outbreak showed several potential chains of transmission, including 3 small family clusters and 2 clusters from evening social gatherings. The timing of these 2 gatherings (Apr. 9 and Apr. 12, 2020) suggests that they were major contributors to the outbreak peak in mid-April 2020. The decrease in cases that occurred after the declaration of the state of emergency, and the absence of any new cases approximately 2 weeks later, indicate that the actions taken during the outbreak (e.g., testing, contact tracing, isolation, quarantine, public messaging, travel restrictions and curfew) were successful in reducing transmission. The results of whole-genome sequencing also support this conclusion: the specific sequence types observed in this outbreak did not transmit further in British Columbia after the outbreak was declared over.

The success in containing transmission in this community was likely a result of the close collaboration between the health authority and 'Namgis First Nation. This collaboration included capitalizing on resources already present in the community, involving community leadership in public health decision-making and asking trusted members of the community to lead communications efforts. Because information came from people with long-standing relationships in the community, public health measures were generally accepted. This was even more notable given that the outbreak occurred at a time when similar measures were not in place elsewhere in British Columbia. Although similar methods have been used by other First Nations communities in Canada to prepare for COVID19 outbreaks, ${ }^{17}$ detailed reports of COVID-19 outbreak response in First Nations communities are lacking.

In addition to the health effects of the outbreak on this First Nations community, it is important to highlight the cultural effects. Like many other First Nations communities across Canada, potlatching, feasting and other ceremonial practices play a vital role in the cultural and spiritual wellbeing of the residents. Living without these gatherings takes a heavy toll, ${ }^{16}$ but their long-lasting effects may not always be evident in the midst of response. Consideration for these effects should be a high priority in any outbreak response, and appropriate supports should be provided. 


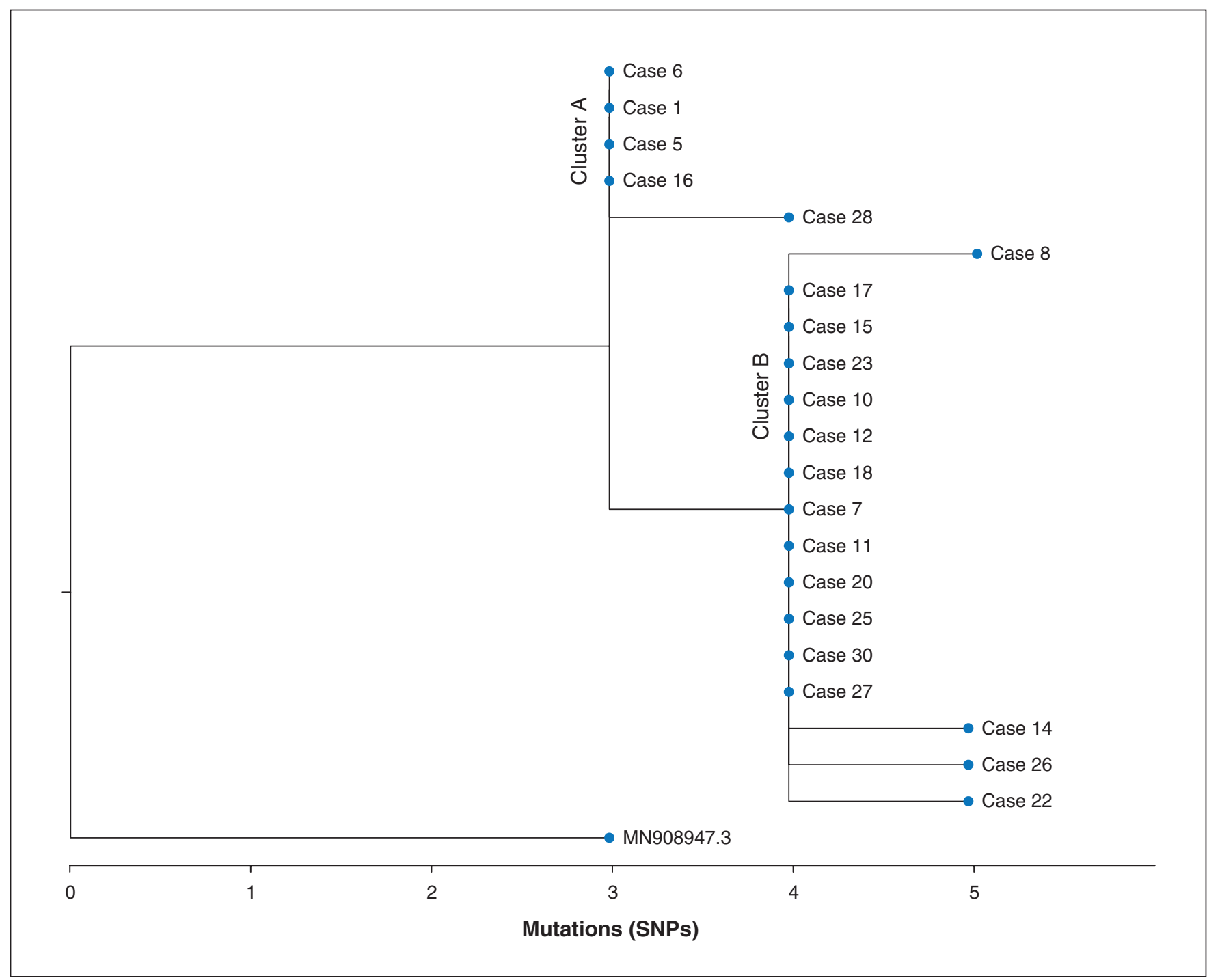

Figure 3: Phylogenetic tree displaying the divergence between SARS-CoV-2 genomes for 21 confirmed COVID-19 cases that generated highquality sequence data and the reference strain MN908947.3 (Wuhan-Hu-1). The scale bar represents the number of mutations, or SNPs, detected in the SARS-CoV-2 genomes from the cases relative to one another and to MN908947.3. Sequences from cases displayed on each vertical line were considered identical. Note: SNP = single nucleotide polymorphism.

This was the first cluster of SARS-CoV-2 to be identified in a remote First Nations community in British Columbia, and one of the first to be identified in Canada. Several lessons can be brought forward for response to similar outbreaks in the future: acknowledging the importance of a community-led response and the role of other providers and responders as partners with the community; having a consistent communitybased emergency coordinator; implementing early and accessible testing; relying on trusted community members to deliver messaging; building on community health care capacity; establishing clear lines of communication with community leaders such as the Chief and council; providing supports to people to allow for successful isolation or quarantine, such as a community-managed alcohol program and social support services; using established relationships with providers from the regional health authority and the First Nations Health Author- ity; and preparing to address logistical challenges, such as the availability of accommodations for isolation and quarantine (within and outside the community), the feasibility of moving cases to and from hospitals, and securing proper technology to facilitate the monitoring of cases and contacts in areas where Internet and cellular service may be unstable.

\section{Limitations}

There are several limitations to consider in this outbreak report. COVID-19 symptoms are nonspecific and selfreported, so it is possible that symptom onset dates were inaccurate, affecting the epidemic curve, the contact tracing and the resulting social network analysis. Because this outbreak occurred early in the pandemic in Canada and in British Columbia, some symptoms were not discussed in the case investigation, such as loss of taste and smell; therefore, we do 
not know the prevalence of these symptoms in the community. Contact tracing data were likely affected by recall bias and social desirability bias. The fact that several components of the social network analysis remained unconnected signifies that contact tracing did not capture all connections. We were not able to identify the pathway by which SARS-CoV-2 entered this remote community, although results of wholegenome sequencing did indicate a potential connection to a superspreading event. Finally, some cases may have been missed because of aforementioned limitations to contact tracing and the focus on symptomatic testing. For this reason, the cumulative incidence in the community may have been higher than what has been reported here.

\section{Conclusion}

This outbreak investigation found a high incidence of SARS$\mathrm{CoV}-2$ infection in a remote First Nations community; transmission occurred primarily through social gatherings and within households. Effective containment was attributed to a community-led response. This outbreak occurred at a time when First Nations communities in Canada had not yet been substantially affected by the COVID-19 pandemic. However, the pandemic's second wave took a major toll on Indigenous communities. We hope that this outbreak report will contribute to the knowledge base for COVID-19 response in First Nations communities and provide important lessons to bring forward to similar outbreaks in other jurisdictions.

\section{References}

1. Marchand-Senécal X, Kozak R, Mubareka S, et al. Diagnosis and management of first case of COVID-19 in Canada: lessons applied from SARS-CoV-1. Clin Infect Dis 2020;71:2207-10.

2. Coronavirus disease (COVID-19): outbreak update. Ottawa: Public Health Agency of Canada; 2021 July 9. Available: www.canada.ca/en/public-health/services/ diseases/2019-novel-coronavirus-infection.html (accessed 2021 Nov. 4).

3. British Columbia COVID-19 dashboard. Vancouver: BC Centre for Disease Control; 2021 Nov. 3. Available: https://experience.arcgis.com/experience/ a6f23959a8b14bfa989e3cda29297ded (accessed 2021 Nov. 4).

4. Eichelberger L, Dev S, Howe T, et al. Implications of inadequate water and sanitation infrastructure for community spread of COVID-19 in remote Alaskan communities. Sci Total Environ 2021;776:145842.

5. Anderson C, Leeson C, Valcourt A, et al. COVID-19 pandemic: implications for First Nations communities in northern Ontario. Univ Toronto Med 7 2021;98:31-4.

6. Erwin C, Aultman J, Harter T, et al. Rural and remote communities: unique ethical issues in the COVID-19 pandemic. Am 7 Bioeth 2020;20:117-20.

7. Census profile, 2016 census. Ottawa: Statistics Canada; 2017, updated 2019 June 18. Available: www12.statcan.gc.ca/census-recensement/2016/dp-pd/prof/ index.cfm?Lang=E (accessed 2021 Jan. 15).

8. Interim guidance: public bealth management of cases and contacts associated with novel coronavirus (COVID-19) in the community. Vancouver: BC Centre for Disease Control; 2021. Available: www.bccdc.ca/resource-gallery/Documents/ Guidelines\%20and\%20Forms/Guidelines\%20and\%20Manuals/Epid/CD\% 20Manual/Chapter\%201\%20-\%20CDC/2019-nCoV-Interim_Guidelines.pdf (accessed 2021 Jan. 15).
9. COVID-19 case report form. Vancouver: BC Centre for Disease Control; 2020 Mar. Available: www.bccdc.ca/Documents/COVID-19_Case_Report_Form. pdf (accessed 2021 Jan. 15).

10. Tyson JR, James P, Stoddart D, et al. Improvements to the ARTIC multiplex PCR method for SARS-CoV-2 genome sequencing using nanopore. bioRxiv 2020 Sept 4 [preprint]. doi: 10.1101/2020.09.04.283077.

11. Rambaut A, Holmes EC, O'Toole Á, et al. A dynamic nomenclature proposal for SARS-CoV-2 lineages to assist genomic epidemiology. Nat Microbiol 2020;5:1403-7.

12. Hadfield J, Megill C, Bell SM, et al. Nextstrain: real-time tracking of pathogen evolution. Bioinformatics 2018;34:4121-3.

13. British Columbia COVID-19 daily situation report, May 26, 2020. Vancouver: BC Centre for Disease Control; 2020 May 26. Available: www.bccdc.ca/Health -Info-Site/Documents/BC_Surveillance_Summary_May_26_2020_FINAL.pdf (accessed 2021 Jan. 15).

14. Banning J. Why are Indigenous communities seeing so few cases of COVID-19? CMA7 2020;192:E993-4.

15. COVID-19 in Canada: modelling update. Ottawa: Health Canada; 2020 updated 2020 June 5. Available: www.canada.ca/en/public-health/services/ publications/diseases-conditions/covid-19-using-data-modelling-inform-public -health-action-april-28-2020.html (accessed 2021 Jul.1).

16. Power T, Wilson D, Best O, et al. COVID-19 and Indigenous Peoples: an imperative for action. 7 Clin Nurs 2020;29:2737-41.

17. Kyoon-Achan G, Write L. Community-based pandemic preparedness: COVID-19 procedures of a Manitoba First Nation community. $7 C S W B$ 2020;5:45-50.

Affiliations: Population Health Assessment, Surveillance, and Epidemiology (Smith), Island Health, Victoria, BC; Canadian Field Epidemiology Program (Smith), Public Health Agency of Canada, Ottawa, Ont.; Department of Public Health \& Preventive Medicine (Enns), Island Health, Courtenay, BC; 'Namgis Health Centre (Cutfeet, James); 'Namgis First Nation (Alfred), Alert Bay, BC; Communicable Disease Program (Lindbeck), Island Health, Courtenay, BC; Public Health Laboratory (Russell), BC Centre for Disease Control, Vancouver, BC.

Contributors: Charmaine Enns, Dan Cutfeet, Nicole James, Jennifer Lindbeck led and/or contributed to outbreak response, were involved in data acquisition and interpretation, and revised the manuscript for intellectual content. Shannon Alfred coordinated emergency response for the community, contributed to data interpretation, and assisted in drafting the manuscript. Courtney Smith analyzed and interpreted all data and drafted the initial manuscript. Shannon Russell conducted and interpreted the whole-genome sequencing analysis and assisted in drafting the manuscript. All authors contributed to the final manuscript, gave final approval for the version to be published, and agree to act as guarantors of the work.

Funding: There was no funding received for this work.

Content licence: This is an Open Access article distributed in accordance with the terms of the Creative Commons Attribution (CC BY-NC-ND 4.0) licence, which permits use, distribution and reproduction in any medium, provided that the original publication is properly cited, the use is noncommercial (i.e., research or educational use), and no modifications or adaptations are made. See: https://creativecommons.org/licenses/by-nc-nd/4.0/

Data sharing: As per the OCAP principles (First Nations principles of ownership, control, access, and possession) any sharing of the data with others requires consultation and approval of the Senior Administrator of 'Namgis First Nation. Please contact courtneyr.smith@canada.ca to facilitate any requests.

Supplemental information: For reviewer comments and the original submission of this manuscript, please see www.cmajopen.ca/content/9/4/ E1073/suppl/DC1 